\title{
Development of a Human Leukocyte Antigen Score to Predict Progression-Free Survival in Head and Neck Squamous Cell Carcinoma Patients
}

\author{
Gunnar Wichmann ${ }^{1,2 *}$, Claudia Lehmann ${ }^{3}$, Cindy Herchenhahn ${ }^{1,4}$, Marlen Kolb ${ }^{1}$, \\ Mathias Hofer ${ }^{1}$, Susanne Wiegand ${ }^{1}$ and Andreas Dietz ${ }^{1,2}$
}

'Clinic for Otorhinolaryngology, Head and Neck Surgery, University Hospital Leipzig, Leipzig, Germany, ${ }^{2}$ LIFE - Leipzig Research Center for Civilization Diseases, University of Leipzig, Leipzig, Germany, ${ }^{3}$ nstitute for Transfusion Medicine, University Hospital Leipzig, Leipzig, Germany, ${ }^{4}$ Clinic for Anesthesiology and Intensive Care, University Hospital Leipzig, Leipzig, Germany

OPEN ACCESS

Edited by: Rasha Abu Eid,

University of Aberdeen,

United Kingdom

Reviewed by:

Panagiotis Balermpas, Goethe University Frankfurt, Germany Naomi Kiyota,

Kobe University Hospital, Japan

*Correspondence:

Gunnar Wichmann gunnar.wichmann@medizin. uni-leipzig.de

Specialty section: This article was submitted to Head and Neck Cancer, a section of the journal

Frontiers in Oncology

Received: 07 March 2018 Accepted: 01 May 2018

Published: 17 May 2018

Citation:

Wichmann G, Lehmann C, Herchenhahn C, Kolb M, Hofer M,

Wiegand S and Dietz A (2018) Development of a Human Leukocyte Antigen Score to Predict

Progression-Free Survival in Head and Neck Squamous Cell Carcinoma Patients. Front. Oncol. 8:168. doi: 10.3389/fonc.2018.00168
Background: In personalized medicine and treatment stratification of head and neck squamous cell carcinoma (HNSCC), the heterogeneous genetic background of patients is not considered. Human leukocyte antigen (HLA) alleles and HLA haplotypes (HLA traits) are linked to development of HNSCC and affect progression-free survival (PFS) of HNSCC patients but most head and neck oncologists are not familiar with HLA typing. Hence, we developed an HLA-score abstracting from complexity of HLA-typing results to facilitate potential use of HLA-associated hazard ratios $(\mathrm{HR})$ for prognostic stratification.

Methods: The HR for PFS of $8 \mathrm{HLA}$ traits shown to be independent predictors $(P l)$ of PFS in a test cohort (TC) of 90 HNSCC patients were used to build the HLA-score based on the natural logarithm (In) of the Pi-associated HR. Crude In-transformed HR of the eight $P i$, alleles $\mathrm{B}^{\star} 13$ (2), $\mathrm{B}^{\star} 35$ (1), $\mathrm{B}^{\star} 51$ (2), DQB1*06 (1), homozygous $\mathrm{Cw}$ (1), homozygous DRB4 (2), and haplotypes $A^{\star} 01 / B^{\star} 08(-6)$ and $B^{\star} 08 / C^{\star} 07$ (4), were summed up to yield the individual patient's HLA-score. Receiver operating characteristic $(R O C)$ and Kaplan-Meier curves were used to proof the suitability of the HLA-score as prognostic marker for PFS. An independent validation cohort (iVC) of 32 patients treated in the larynx-organ preservation trial DeLOS-II was utilized for validation.

Results: The individual HLA-scores (range -2 to 6) in TC classified HNSCC patients regarding PFS. ROC analysis (area under the curve $=0.750,95 \% \mathrm{Cl} 0.665-0.836$; $P=0.0000034)$ demonstrated an optimum cutoff for the HLA-score at $0.5(97.9 \%$ sensitivity, 34.7\% specificity), and 70/90 patients in TC with HLA-score $>0$ had significant reduced PFS $(P=0.001)$. Applying the same classifier (HLA-score $>0$ ) confirmed these findings in the IVC revealing reduced PFS of $25 / 32$ patients $(P=0.040)$.

Conclusion: HLA traits constitute critical Pi. Considering the HLA-score may potentially facilitate the use of genetic information from HLA typing for prognostic stratification, e.g., within clinical trials.

Keywords: head and neck squamous cell carcinoma, head and neck cancer, human leukocyte antigen, human leukocyte antigen haplotype, progression-free survival, larynx-organ preservation trial, biomarker score, independent predictor 


\section{INTRODUCTION}

The genetic background of head and neck squamous cell carcinoma (HNSCC) patients and a potential genetic predisposition for development of HNSCC and relapse after successfully applied curative treatment are almost completely ignored. By contrast, somatic mutations, epigenetic changes and divergent gene-expression patterns in HNSCC and tumor-infiltrating lymphocytes present in the tumor are assessed as prognostic and predictive biomarkers (1-8). Main risk factors for carcinogenesis of HNSCC are tobacco and/or alcohol consumption, but the risk is modified by genetic polymorphisms (8-13). In addition, infection with oncogenic subtypes of the human papillomavirus (HPV), especially HPV16 and other high-risk subtypes, is etiologically involved in development of HNSCC of the oropharynx (14). HPV-related HNSCC are characterized by distinct molecular features $(2-6,8,15)$. High level tobacco smoking, daily alcohol drinking as well as HPV-related carcinogenesis and especially their simultaneous presence are accompanied by immunotoxicity, genotoxicity, mutagenesis plus increased expression of cytokines and growth factors and exhausted immune response resulting in loss of proliferation control (16). Therefore, and because of the multitude of studies demonstrating the association of these risk factors with HNSCC and outcome, the reasons for developing HNSCC and also relapse after initially curative treatment appear to be obvious. Consequently, and in contrast to many other cancer entities, both occurrence and relapse of HNSCC are mostly seen as attributable to the patient's lifestyle. However, tobacco smoking and alcohol together explain only $73 \%$ of upper aerodigestive tract cancer incidence totally ranging from below $50 \%$ in HNSCC-affected women to about $85 \%$ in laryngeal and hypopharyngeal HNSCC in men (12). The risk for development of HNSCC is increased by genetic variants in genes encoding enzymes involved in DNA repair or metabolism of alcohol $(9-11,13)$; Fanconi anemia patients have a 700 -fold increased risk for HNSCC and high rate of relapses $(17,18)$. Moreover, research implicated that polymorphisms in cytokine genes and members of the immunoglobulin supergene family including human leukocyte antigens (HLAs) are involved in either improved or impaired ability to control somatic mutations by adequate immune responses and maintenance of immune surveillance: we recently demonstrated in a German cohort of white Caucasian genetic descent that polymorphisms in HLA, in particular HLA-B antigens and homozygosity in HLA-Cw and DRB4, are associated with increased risk for HNSCC (19). Moreover, the highly frequent disruption of functionally coupled HLA antigens (haplotypes) and presence of uncommon haplotypes in a significant proportion of patients are linked not only to development of HNSCC but even more to reduced progressionfree survival (PFS) independent of lifestyle-associated risk factors (19). By contrast, compared with healthy blood donors, some HLA traits are detected in HNSCC in lower frequency. The fewer carriers of such haplotypes have improved PFS, whereas those that are over-represented did not. Hence, genetic heterogeneity seems to account for altered risk of developing HNSCC but also PFS (19). The hazard ratios (HR) for PFS of $B^{\star} 13, B^{\star} 35, B^{\star} 51$, HLA-DQB1 ${ }^{\star} 06$, homozygous $\mathrm{Cw}$ and DRB4, and the haplotypes
$\mathrm{A}^{\star} 01 / \mathrm{B}^{\star} 08$ and $\mathrm{B}^{\star} 08 / \mathrm{C}^{\star} 07$ were stably significant independent predictors $(P i)$ in multivariate analyses (19). They also may be considered as prognostic factors in comparative analyses, e.g., in clinical trials.

However, the use of raw low-resolution tissue-typing results to assess the risk for PFS according to presence of a particular HLA trait and an individual risk attributed to any risk factor including $P i$ appears to be not very useful to estimate the individual patient's risk for relapse in clinical routine. For the latter purpose, an abstraction from individual polymorphisms could be helpful. Very desirable but not at hand is an easy-to-use way to assess the individual patient's risk attributable to his/her HLA type. As the aggregation of independent risk factors in a score offers a way to abstract from individual risk factors by summarizing only their (potential) impact as $P i(20)$, we had the hypothesis that HR for PFS may be useful to construct an HLA-score. Therefore, we newly defined an HLA-score based on published HR from our recent findings in a test cohort (TC) (19). Here, we aim to verify a potential impact of the scored HLA traits on PFS of HNSCC and to particularly elucidate, if this HLA-score reliably predicts outcome differences in the context of clinical trials. Hence, low-resolution HLA typing of leukocytes from an independent validation cohort (iVC), 32 laryngeal/hypopharyngeal HNSCC patients treated in the DeLOS-II larynx-organ preservation trial $(20,21)$, was performed. Related to their HLA-scoring the PFS in the iVC was analyzed and confirmed the prognostic value of the HLA-score.

\section{MATERIALS AND METHODS}

\section{HNSCC Patients and Study Population}

This study was carried out in accordance with the recommendations of the guidelines of the ethics committee of the Medical Faculty of the University Leipzig. The protocol was approved by the ethics committee of the Medical Faculty of the University Leipzig (vote no. 201-10-12072010 and no. 202-10-12072010). All subjects gave written informed consent in accordance with the Declaration of Helsinki.

\section{Test Cohort}

Blood samples were from histopathologic confirmed HNSCC patients (Table 1) of white Caucasian genetic descent diagnosed and treated between 08/2010 and 05/2011 at the ENT Department of the University Hospital Leipzig. 12 of the 90 patients in the TC were treated in the larynx-organ preservation trial DeLOS-II (21) (NCT00508664; advanced HNSCC of the hypopharynx or larynx receiving induction chemotherapy followed by radiotherapy \pm cetuximab; $n=12$ ). For HLA typing, genomic DNA was isolated using the salting out procedure (22) from leukocytes of blood samples. Low-resolution DNA-typing was performed using PCR-SSP for HLA-A, B, Cw, HLA-DRB1, DRB3/4/5, and DQB1 as described elsewhere (18).

\section{Independent Validation Cohort}

DNA samples from peripheral blood of additional 32 of the 52 LHSCC patients treated in the DeLOS-II trial in Leipzig 
TABLE 1 | Main characteristics of the head and neck squamous cell carcinoma patients of the test cohort (TC; $N=90$ ) and independent validation cohort (iVC; $N=32$ ) investigated.

\begin{tabular}{|c|c|c|c|c|c|c|}
\hline \multirow[b]{2}{*}{ Covariate } & \multirow[b]{2}{*}{ Category } & \multicolumn{2}{|c|}{ TC } & \multicolumn{2}{|r|}{ ivc } & \multirow[b]{2}{*}{$P$ value } \\
\hline & & $n$ & (\%) & $n$ & (\%) & \\
\hline \multirow[t]{2}{*}{ Sex } & Female & 12 & (13.3) & 5 & (15.6) & 0.748 \\
\hline & Male & 78 & (86.7) & 27 & (84.4) & \\
\hline \multirow[t]{6}{*}{ Localization } & Oropharynx & 28 & (31.1) & 0 & $(0.0)$ & $<0.001$ \\
\hline & Other & 62 & (68.9) & 32 & (100.0) & \\
\hline & Oropharynx & 28 & (31.1) & 0 & $(0.0)$ & $<0.001$ \\
\hline & Hypopharynx & 20 & (22.2) & 19 & (59.4) & \\
\hline & Larynx & 24 & $(26.7)$ & 13 & (40.6) & \\
\hline & Other & 18 & (20.0) & 0 & $(0.0)$ & \\
\hline \multirow[t]{6}{*}{ T category } & Tx & 1 & $(1.1)$ & 0 & $(0.0)$ & 0.025 \\
\hline & $\mathrm{T} 1$ & 13 & (14.4) & 0 & $(0.0)$ & \\
\hline & T2 & 21 & (23.3) & 3 & $(9.4)$ & \\
\hline & T3 & 21 & (23.3) & 15 & (46.9) & \\
\hline & $\mathrm{T} 4 \mathrm{a}$ & 32 & (35.6) & 14 & (43.8) & \\
\hline & $\mathrm{T} 4 \mathrm{~b}$ & 2 & $(2.2)$ & 0 & $(0.0)$ & \\
\hline \multirow[t]{6}{*}{$\mathrm{N}$ category } & No & 32 & (35.6) & 3 & $(9.4)$ & 0.010 \\
\hline & N1 & 7 & $(7.8)$ & 1 & $(3.1)$ & \\
\hline & $\mathrm{N} 2 \mathrm{a}$ & 5 & (5.6) & 0 & $(0.0)$ & \\
\hline & N2b & 22 & (24.4) & 13 & (40.6) & \\
\hline & $\mathrm{N} 2 \mathrm{C}$ & 9 & (10.0) & 14 & (43.8) & \\
\hline & N3 & 5 & $(5.6)$ & 1 & $(3.1)$ & \\
\hline \multirow[t]{2}{*}{ N category } & No & 32 & (35.6) & 3 & $(9.4)$ & 0.005 \\
\hline & $\mathrm{N}+$ & 58 & $(64.4)$ & 29 & $(90.6)$ & \\
\hline \multirow[t]{2}{*}{ M category } & MO & 87 & $(96.7)$ & 32 & $(100.0)$ & \\
\hline & M1 & 3 & (3.3) & 0 & $(0.0)$ & \\
\hline \multirow[t]{6}{*}{ Stage } & UICC I & 8 & (8.9) & 0 & $(0.0)$ & 0.055 \\
\hline & UICC II & 11 & (12.2) & 0 & $(0.0)$ & \\
\hline & UICC III & 9 & (10.0) & 4 & (12.5) & \\
\hline & UICC IVA & 53 & (58.9) & 27 & $(84.4)$ & \\
\hline & UICC IVB & 6 & $(6.7)$ & 1 & $(3.1)$ & \\
\hline & UICC IVC & 3 & (3.3) & 0 & $(0.0)$ & \\
\hline \multirow[t]{2}{*}{ Stage } & Early & 19 & $(21.1)$ & 0 & $(0.0)$ & 0.006 \\
\hline & Advanced & 71 & (78.9) & 32 & (100.0) & \\
\hline \multirow{6}{*}{$\begin{array}{l}\text { Human } \\
\text { papillomavirus } \\
\text { (HPV) status }\end{array}$} & High-risk HPV-DNA+ & 17 & (18.9) & 0 & $(0.0)$ & 0.008 \\
\hline & High-risk HPV-DNA- & & 73 & 32 & (100.0) & \\
\hline & HPV16-DNA+ & 13 & (14.4) & 0 & $(0.0)$ & 0.023 \\
\hline & HPV16-DNA- & 77 & (85.6) & 32 & $(100.0)$ & \\
\hline & HPV16-DNA + RNA+ & 8 & (8.9) & 0 & $(0.0)$ & 0.081 \\
\hline & HPV16 RNA- & 82 & $(91.1)$ & 32 & $(100.0)$ & \\
\hline \multirow{10}{*}{$\begin{array}{l}\text { Tobacco } \\
\text { smoking } \\
\text { behavior }\end{array}$} & Non-smoker & 19 & $(21.1)$ & 0 & $(0.0)$ & 0.004 \\
\hline & Smoker & 70 & $(77.8)$ & 32 & $(100.0)$ & \\
\hline & Missing & 1 & $(1.1)$ & 0 & $(0.0)$ & \\
\hline & Non-smoker & 19 & (21.1) & 0 & $(0.0)$ & 0.046 \\
\hline & <10 pack years & 2 & $(2.2)$ & 2 & (6.3) & \\
\hline & $10<20$ pack years & 5 & (5.6) & 5 & (15.6) & \\
\hline & $20<30$ pack years & 8 & (8.9) & 4 & $(12.5)$ & \\
\hline & $30<40$ pack years & 26 & (28.9) & 9 & $(28.1)$ & \\
\hline & $\geq 40$ pack years & 29 & $(32.2)$ & 12 & $(37.5)$ & \\
\hline & Missing & 1 & $(1.1)$ & 0 & $(0.0)$ & \\
\hline \multirow{3}{*}{$\begin{array}{l}\text { Alcohol } \\
\text { consumption }\end{array}$} & No & 9 & $(10.0)$ & 1 & $(3.4)$ & 0.218 \\
\hline & Yes & 80 & (88.9) & 31 & (96.6) & \\
\hline & Missing & 1 & $(1.1)$ & 0 & $(0.0)$ & \\
\hline \multirow{5}{*}{$\begin{array}{l}\text { Alcohol } \\
\text { consumption } \\
\text { category }\end{array}$} & 0 & 10 & $(11.1)$ & 1 & $(3.4)$ & 0.455 \\
\hline & $>0<30 \mathrm{~g} /$ day & 31 & (34.4) & 11 & $(34.4)$ & \\
\hline & $30<60$ g/day & 24 & $(26.7)$ & 8 & $(25.0)$ & \\
\hline & $\geq 60 \mathrm{~g} /$ day & 24 & $(26.7)$ & 12 & $(37.5)$ & \\
\hline & Missing & 1 & (1.1) & 0 & $(0.0)$ & \\
\hline
\end{tabular}

TABLE 1 | Continued

\begin{tabular}{|c|c|c|c|c|c|c|}
\hline \multirow[b]{2}{*}{ Covariate } & \multirow[b]{2}{*}{ Category } & \multicolumn{2}{|c|}{$\mathrm{TC}$} & \multicolumn{2}{|r|}{ iVC } & \multirow[b]{2}{*}{$P$ value } \\
\hline & & $n$ & (\%) & $n$ & (\%) & \\
\hline \multirow[t]{8}{*}{ Therapy } & Surgery (Op) & 19 & $(21.1)$ & 0 & $(0.0)$ & $<0.001$ \\
\hline & Radiotherapy (RT) & 6 & $(6.7)$ & 0 & $(0.0)$ & \\
\hline & Op + PORT & 19 & $(21.1)$ & 0 & $(0.0)$ & \\
\hline & Op + PORChT & 16 & (17.8) & 0 & $(0.0)$ & \\
\hline & Primary concurrent RChT & 3 & (3.3) & 0 & $(0.0)$ & \\
\hline & DeLOS-II (IC + RT) & 12 & (13.3) & 32 & $(100.0)$ & \\
\hline & $\mathrm{IC}+\mathrm{Op}+\mathrm{PORT}$ & 13 & (14.4) & 0 & $(0.0)$ & \\
\hline & Best supportive care (BSC) & 2 & $(2.2)$ & 0 & $(0.0)$ & \\
\hline \multirow{15}{*}{$\begin{array}{l}\text { Therapies } \\
\text { applied }\end{array}$} & Monomodal & 25 & $(27.8)$ & 0 & $(0.0)$ & 0.002 \\
\hline & Multimodal & 63 & $(70.0)$ & 32 & (100.0) & \\
\hline & BSC & 2 & $(2.2)$ & 0 & $(0.0)$ & \\
\hline & Received Op & 68 & (75.6) & 5 & $(15.6)$ & $<0.001$ \\
\hline & No & 22 & $(24.4)$ & 27 & $(84.4)$ & \\
\hline & Received RT & 69 & $(76.7)$ & 32 & $(100.0)$ & 0.003 \\
\hline & No & 21 & (23.3) & 0 & $(0.0)$ & \\
\hline & $\begin{array}{l}\text { Received chemotherapy } \\
(\mathrm{ChT})\end{array}$ & 44 & $(48.9)$ & 32 & $(100.0)$ & $<0.001$ \\
\hline & No & 46 & $(51.1)$ & 0 & $(0.0)$ & \\
\hline & Received Op + RT & 49 & $(54.4)$ & 5 & $(15.6)$ & $<0.001$ \\
\hline & No & 41 & $(45.6)$ & 27 & $(84.4)$ & \\
\hline & Received ChT + RT & 44 & $(48.9)$ & 32 & $(100.0)$ & $<0.001$ \\
\hline & No & 46 & $(51.1)$ & 0 & $(0.0)$ & \\
\hline & Received Op + ChT + RT & 30 & (33.3) & 5 & $(15.6)$ & 0.057 \\
\hline & No & 60 & $(66.7)$ & 27 & $(84.4)$ & \\
\hline
\end{tabular}

$(20,21)$ underwent low-resolution HLA typing utilizing BMT OneLambda SSO-typing kits for HLA-A, B, Cw, DQ, and DR according to the manufacturer's instructions. Blood samples of eight DeLOS-II patients were not available as three patients were already deceased, two patients were reluctant to participate in the study and donate blood for genotyping, and three patients were lost to follow-up. Avoiding overlap with the 12 DeLOS-II patients in TC, the iVC consists of $N=32$ independent samples. Presence of the eight HLA traits constituting Pi of PFS in the TC was assessed as described (19).

\section{Development of the HLA-Score}

The recently published HR of HLA traits detected in the TC that consistently remained significant $P i$ for PFS after bootstrapping with 1,000 iterations (19) were used to build the HLA-score. This data set from the TC (19) fulfils all prerequisites to classify the patients based on a score combining up to eight independent predictors $\left(n=90>64=8^{2}\right)$. The HR for each of the eight HLA traits was transformed into its natural logarithm, ln HR (Table 2). Absence of a genetic predictor scored 0 , while its presence scored with the crude $\ln$ HR denoted in brackets: HLA-B ${ }^{\star} 13$ (2), HLA-B ${ }^{\star} 35$ (1), HLA-B ${ }^{\star} 51$ (2), HLA-DQB1 ${ }^{\star} 06$ (1), homozygous HLA-Cw (1), homozygous HLA-DRB4 (2), haplotype $A^{\star} 01 / B^{\star} 08$ $(-6)$, haplotype $B^{\star} 08 / C^{\star} 07(4)$. These scores were summed up to build the HLA-score of the individual patient.

\section{Statistical Analysis}

Pearson's chi-square $\left(\chi^{2}\right)$ test for contingency tables was used to analyze differences between groups. PFS was chosen as end point in survival analyses and measured from registration date until 
TABLE 2 | Significant independent predictors for progression-free survival of head and neck squamous cell carcinoma patients according to published data from the multivariate Cox regression model (18) used to define the human leukocyte antigen (HLA) score.

\begin{tabular}{|c|c|c|c|c|c|c|c|c|c|}
\hline \multirow[t]{2}{*}{ Covariates } & \multicolumn{5}{|c|}{$\mathrm{TC}$} & \multicolumn{4}{|c|}{ Validation cohort } \\
\hline & HR & $(95 \% \mathrm{Cl})$ & $\begin{array}{c}P \text { value } \\
\text { (2-sided) }\end{array}$ & $\begin{array}{c}P \text { value (2-sided; } \\
\text { in bootstrapping) }\end{array}$ & $n(\%)$ & $n(\%)$ & $\begin{array}{c}P \text { value } \\
\left(2-\text { sided }^{\mathrm{b}}\right.\end{array}$ & In HR & HLA-score \\
\hline HLA-B*13 & 7.460 & $(2.212-25.16)$ & 0.0012 & 0.025 & $11(12.2)$ & $4(12.5)$ & 0.967 & 2.010 & 2 \\
\hline HLA-B*35 & 2.630 & $(1.543-4.485)$ & 0.0004 & 0.017 & $15(16.7)$ & $5(15.6)$ & 0.891 & 0.967 & 1 \\
\hline HLA-B*51 & 9.278 & (2.270-37.92) & 0.0019 & 0.015 & $8(8.9)$ & $4(12.5)$ & 0.556 & 2.228 & 2 \\
\hline HLA-DQB1*06 & 1.890 & $(1.152-3.101)$ & 0.0117 & 0.037 & $40(44.4)$ & $10(31.3)$ & 0.192 & 0.637 & 1 \\
\hline Homozygous HLA-Cw & 4.292 & $(1.864-9.888)$ & 0.0006 & 0.001 & $27(30.0)$ & $10(31.3)$ & 0.895 & 1.457 & 1 \\
\hline Homozygous HLA-DRB4 & 9.513 & $(2.787-32.47)$ & 0.0003 & 0.007 & $10(11.1)$ & 7 (21.9) & 0.131 & 2.253 & 2 \\
\hline Haplotype A*01/B*08 & 0.003 & (0.000-0.054) & 0.000056 & 0.003 & $11(12.2)$ & $3(9.4)$ & 0.664 & -5.809 & -6 \\
\hline Haplotype $B^{\star} 08 / C^{\star} 07$ & 74.856 & $(12.58-445.4)$ & 0.000002 & 0.001 & $16(17.8)$ & $5(15.6)$ & 0.782 & 4.316 & 4 \\
\hline
\end{tabular}

Hazard ratio (HR plus 95\% Cl in brackets) and the associated 2-sided $P$ value, plus $P$ values from bootstrapping applying 1,000 iterations are shown accompanied by their frequencies in the test cohort (TC) $(N=90)$ and independent validation cohort $(N=32)$.

The HLA-scores (highlighted bold) for the individual respective HLA trait are derived from the natural logarithm (In)-transformed HR.

aBootstrapping using 1,000 iterations.

${ }^{b}$ Comparison of frequencies in TC versus iVC using Pearson's $\chi^{2}$ test.

date of either cancer-related death or relapse, censoring patients without any malignancy at last follow-up, or deaths not related to cancer. As relapse were considered local recurrence, lymph node or distant metastasis, or second primaries. PFS was analyzed using Kaplan-Meier curves (SPSS version 20, IBM Corporation, Armonk, NY, USA) applying log-rank tests (Mantel-Cox). Receiver operating characteristics (ROC) for PFS versus HLAscore were assessed using SPSS. $P$ values $<0.05$ from two-sided tests were considered significant.

\section{RESULTS}

Table 1 shows the characteristics of both cohorts. Some significant inequalities in risk-factor distributions were detected. According to the study protocol of the DeLOS-II trial all 32 LHSCC patients of the iVC were of advanced stage (UICC III, IV) with higher $\mathrm{T}$ and $\mathrm{N}$ categories. They were exclusively smokers and not HPV-related $(P<0.01)$, and based on the per-protocol treatment in DeLOS-II $(20,21)$ their treatment differed significantly from the TC (Table 1). Despite the lower case number in iVC, a comparable distribution and frequency of the HLA traits included in the HLA-score was observed and found being without any significant difference (Table 2).

The individual patient's HLA-score as defined by the sum of the crude ln-transformed HR of the eight HLA traits resulted in a comparable distribution of HLA-scores $(P=0.683)$. These scores were found in cohorts TC/iVC: $-2(n=3 / 2 ; 3.3 / 6.3 \%)$, $-1(n=5 / 1 ; 5.6 / 3.1 \%), 0(n=12 / 4 ; 13.3 / 12.5 \%), 1(n=29 / 6$; $32.2 / 18.8 \%), 2(n=22 / 11 ; 24.4 / 34.4 \%), 3$ ( $n=9 / 4 ; 10 / 12.5 \%), 4$ $(n=4 / 0 ; 4.4 / 0 \%), 5(n=3 / 2 ; 3.3 / 6.3 \%)$, and $6(n=3 / 2 ; 3.3 / 6.3 \%)$.

Figure 1A shows Kaplan-Meier analyses of PFS in the TC according to HLA-score quartiles. The PFS of HNSCC patients is inversely correlated with HLA-score quartiles. Applying the log-rank test, a significant different PFS of patients of the TC was observed $(P<0.00001)$.

Receiver operating characteristic analyses revealed a significant area under the curve (AUC) for PFS event versus HLAscore $(\mathrm{AUC}=0.750,95 \%$ CI $0.665-0.836 ; P=0.0000034)$ with
HLA-score 0.5 being the optimum cutoff for discrimination of HNSCC patients with good versus impaired PFS in the TC (Figure 1B). Binary classification of TC patients applying this cutoff offers $34.7 \%$ specificity and $97.9 \%$ sensitivity corresponding to a negative predictive value (NPV) of $94.7 \%$ of the HLAscore $\leq 0$ for relapse or cancer-related death.

Kaplan-Meier analyses confirmed the optimal binary classification into groups of patients without HLA-attributable risk (HLA-score $\leq 0 ; n=20$ ) versus those at risk (HLA-score $>0$; $n=70)$ and achieved in the TC the most significant discrimination between groups with deviating PFS ( $P<0.001$; Figure 1C).

The impact of the HLA-score on PFS was further analyzed in the iVC. The HLA-score $\leq 0.5$ had in this cohort an NPV of $100 \%$. In full agreement, and despite the smaller sample size of $N=32$, the HLA-score $\leq 0$ and $>0$ exactly predicted in KaplanMeier curves either prolonged or shortened PFS, respectively, of the iVC patients $(P=0.040$; Figure 1D).

\section{DISCUSSION}

The TC of 90 HNSCC patients demonstrated altered frequencies of HLA antigens and two-locus haplotypes, as well as high frequent homozygosis in Cw and DRB4. The eight HLA traits identified as stable $P i$ respective to a significant impact on PFS of HNSCC patients can be combined to build an HLA-score. As shown here for the first time, the HLA-score of an HNSCC patient, which is the sum of crude ln-transformed HR of the eight HLA traits, is inversely correlated with the PFS and is a Pi. Multivariate analysis in the TC revealed significant altered PFS in carriers of homozygous Cw and DRB4, four HLA-B alleles, and two haplotypes (19). The in Wichmann et al., 2017 (19) applied Cox model (due to inclusion of HLA traits) no longer included $\mathrm{N}$ category $\mathrm{N} 0$ versus $\mathrm{N}+(P=0.520)$, alcohol consumption $(P=0.541)$, sex $(P=0.118)$, and age at diagnosis $(P=0.253)$, as these covariates lowered its overall significance (19). The here newly established HLA-score defines groups of HNSCC patients with significant different PFS independent from these "classical" risk factors for HNSCC (19). This may be seen also in the context 

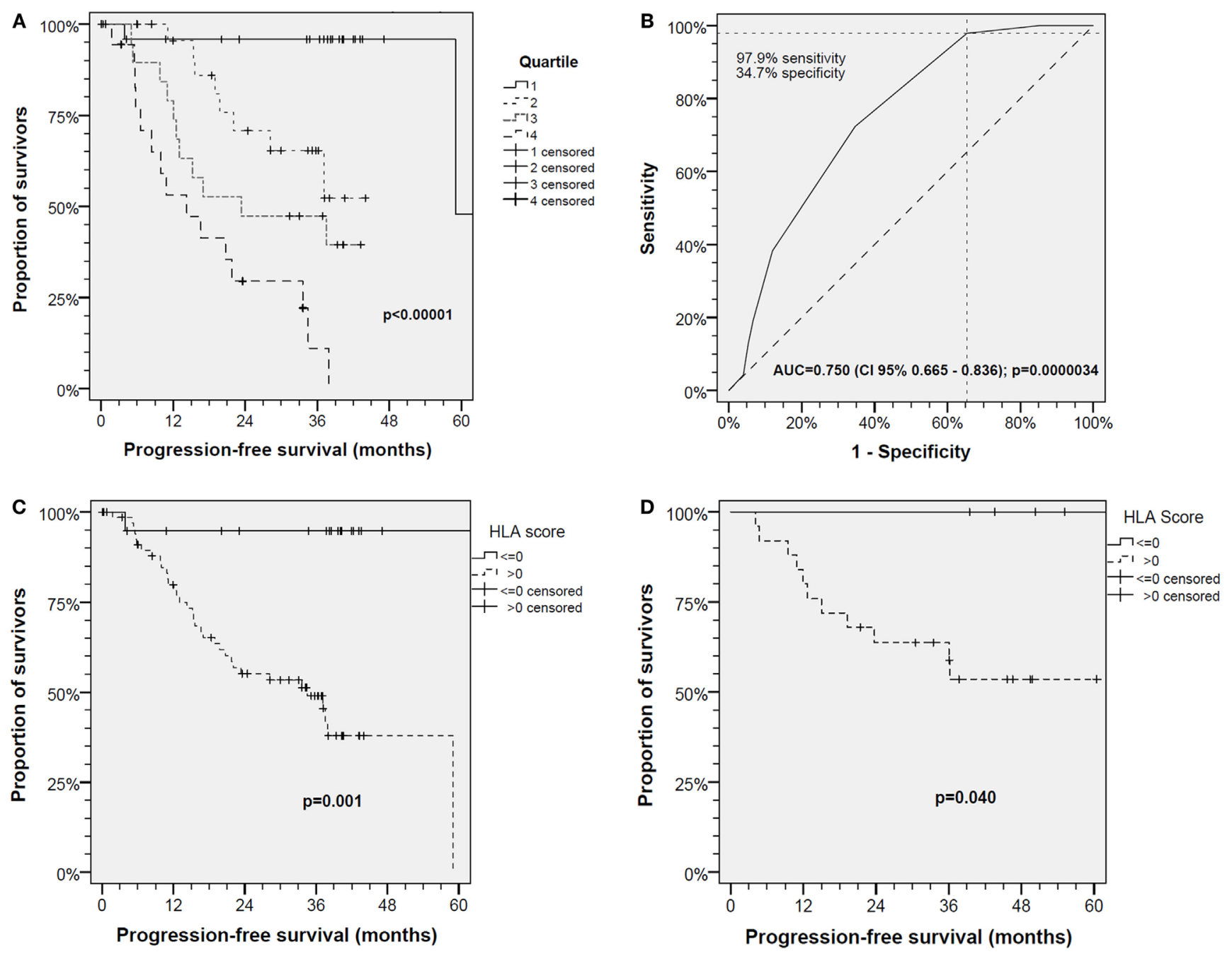

FIGURE 1 | The human leukocyte antigen (HLA)-score is an independent predictor of progression-free survival (PFS) in head and neck squamous cell carcinoma (HNSCC) patients. (A) PFS in the $N=90$ HNSCC patients of the test cohort (TC) respective to HLA-score quartiles; (B) receiver operating characteristics for PFS event versus HLA-score demonstrate high significance and optimum classification with $97.9 \%$ sensitivity and $34.7 \%$ specificity at a cutoff of 0.5 in the TC; (C) PFS in the TC of $N=90 \mathrm{HNSCC}$ patients classified according to cutoff 0.5 ; (D) PFS in the $N=32$ patients of the independent validation cohort classified according to cutoff 0.5 confirms the findings in the TC.

of unexplained risk for development of HNSCC outside the main risk factors tobacco and alcohol (12).

The cutoff 0.5 allowed for discrimination of patient groups with different PFS according to their HLA-score also in the iVC. Patients with HLA-score $>0$ had a significant higher risk for relapse $(P=0.040)$ compared with patients with HLA-score $\leq 0$ who were without event and confirmed the findings in the TC (Figure 1D). This suggests that the HLA-score is potentially able to summarize the HR of HLA traits in a single measure that inversely correlates with the PFS and may be useful as stratification factor for clinical trials, observational studies or in personalized medicine.

Taken together, the results obtained by applying the HLA-score demonstrate the possibility that HLA traits are able to explain at least partially the high level of variance in outcome within clinical trials as demonstrated by the 32 patients of the iVC (Figure 1D) treated in the DeLOS-II larynx-organ preservation trial $(20,21)$. The distribution of HLA traits between study arms therefore may affect the outcome in clinical trials. Frequencies of antigens and haplotypes shown to be $P i$ in the TC range between a few percent (4.4 up to $23.9 \%$ ), and homozygosity in Cw and DRB4 was detected in 30.0 and $11.1 \%$, respectively (19). As effective randomization regarding multiple risk factors each of them individually present in low frequency requires prohibitive high case numbers, unevenly distributed HLA traits could explain failure or irreproducibility of clinical trials even if higher case numbers are compared. This will occur as long as the multitude of low frequent $P i$ is not considered in stratification before randomization. The HLA-score may allow for overcoming this issue by assessment of the risk associated with particular HLA traits which are $P i$.

What are the reasons behind the effect of HLA traits and the HLA-score on PFS of HNSCC? In HNSCC oncology, the 
exposure to tobacco smoke and alcohol are the dominant and most accepted risk factors for development and relapse of HNSCC as they are observed in high frequency and shown to be causative for mutations, e.g., in oncogenes or inactivation of tumor-suppressor genes but also resistance to immune surveillance (23). Research showed a broad spectrum of mutated genes and affected signaling pathways in HNSCC $(2-5,8)$. However, most sporadic somatic mutations or viral infections potentially causing neoplastic transformation are controlled by the immune system. Consequently, altered peptides derived from mutated or viral proteins are in majority efficiently presented to CTL $\left(\mathrm{CD}^{+}\right.$cytotoxic $\mathrm{T}$ lymphocytes) via HLA-A, B, and Cw enabling antigen-specific CTL to bind and delete cancerous cells expressing aberrant or viral proteins. Obviously, these mechanisms work well in most people but not so well in most HNSCC patients. This may be caused either by inadequate binding of T cell receptors to MHC:peptide complexes or incapability of the HLA-proteins to process tumorassociated antigens (TAA) by proteolytic cleavage and to bind particular TTA-derived peptides. Besides the often observed MHC class I loss in $\operatorname{HNSCC}(24,25)$ allowing immune escape, there are indeed huge differences between certain HLA antigens to bind TAA-derived peptides (26). HLA antigens combined in particular haplotypes may have gaps in the capability to bind and present particular TAA-derived peptides. Consequently, the HLA antigens and haplotypes differ in the probability to appropriately activate T cells and get rid of cancer cells expressing peculiar proteins (27). Such impaired competence to maintain immune surveillance is suggested to be related to particular HLA alleles (and haplotype combinations) to efficiently bind and present altered peptides (26) and trigger deletion of the mutated cell by CTL (27). This may cause varying numbers of tumor-infiltrating $\mathrm{CD}^{+} \mathrm{T}$ cells within HNSCC as shown recently $(5,8,28)$.

The HLA-score reliably predicts PFS of HNSCC patients. Even without any clinical information, the stratification using the HLA-score distinguished HNSCC with significant deviating PFS in TC and iVC (Figure 1). We detected significant superior outcome in patients with HLA-score $\leq 0$ with strongly improved relative risk and odds ratios by optimum classification identified in ROC analyses (Figure 1B). It might be important that the HLA-score is able to indicate significant outcome differences even within the small sample of 32 patients in the iVC which moreover had a huge heterogeneity in many response-associated parameters (20). This is even more important as the case number of 32 is much too low to demonstrate significant outcome differences between LHSCC patients related to the treatment, e.g., arm A versus B (20).

Within our study, we noticed that sole presence of one of the eight $P i$ constituting the HLA-score not necessarily predicts the outcome regarding reduced PFS. This is in full agreement with the general explanations by Powers (29) who stated that the directions of implications are not in general dependent: if $\mathrm{Pi}$ is one of several independent possible causes of the condition $R$ (i.e., PFS), $P i \rightarrow R$ is strong, but $R \rightarrow P i$ is in general weak for any specific $P i$. If $P i$ is one of several contributing factors to the condition $R, P i \rightarrow R$ is weak for any single $P i$, but $R \rightarrow P i$ is strong (29). Regarding our HLA-score, this means that each of the eight included independent predictors $P i$ for the condition $R$ (event regarding PFS) alone is weak in explaining the outcome
(PFS), and presence of a particular $P i$ (either alleles HLA-B ${ }^{\star} 13$, $\mathrm{B}^{\star} 35, \mathrm{~B}^{\star} 51, \mathrm{DQB} 1^{\star} 06$, haplotypes $\mathrm{B}^{\star} 08 / \mathrm{Cw}^{\star} 07$ and $\mathrm{A}^{\star} 01 / \mathrm{B}^{\star} 08$, homozygous DRB4 or Cw) stands not against good outcome in general. Vice versa, the HLA-score $\leq 0$ summarizing the eight $P i$ strongly predicts superior outcome, and the outcome explains the high predictive value of the HLA-score (Figure 1).

Our study has some limitations. The impossibility of familial HLA typing due to unavailability of DNA from parents of our patients allowed only for evaluation of estimated haplotypes (phenotypic combinations). However, this is the appropriate and most-often used method for analysis of HLA haplotypes and disease associations (30-32). The small sample size in the iVC might have caused reduced power to replicate the findings in the TC regarding particular HLA traits which are present in frequencies below $10 \%$, e.g., HLA-B alleles and estimated haplotypes, on PFS. However, the abovementioned causality according to $P i \rightarrow R$ applies (29).

Nevertheless, the recently detected HLA-trait dependence of PFS and the possibility to use the newly developed HLA-score requests further investigations to provide proof of reproducibility within cohorts of different genetic background. The possibility exists that the HLA-score can apply only to patients of white Caucasian genetic descent as particular alleles/antigens may behave in a different way when present in a different genetic environment. Some similarities may exist (e.g., the role of homozygosity in particular of $\mathrm{Cw}$ respective to an impaired prognosis), but ethnicity-dependent differences in distribution of particular HLA antigens/alleles and the association of varying haplotypes, e.g., with autoimmune diseases suggest the existence of such inequalities. They might have been at least partially responsible for unresolved issues of varying outcome especially observed in multinational clinical trials. For instance, in the SPECTRUM trial a huge difference was seen outcome of patients treated in Europe and in the U.S. of America compared with patients from the Asian-Pacific region (33) which we expected being at least partially related to genetic heterogeneity in HLA traits of the ethnicities. Therefore, it would be very welcome if HLA typing at least at the low-resolution level is performed within clinical trials including HNSCC patients of other ethnicities to elucidate HLA traits with the potential to differently affect outcome. This should clarify if patient stratification according to HLA traits is possible in multinational trials and if the here presented HLA-score is able to improve reproducibility in future clinical trials.

The identification of a subgroup of patients based on the HLA-score in both cohorts with uniquely superior PFS argues for consideration of HLA traits as stratification factors in head and neck oncology.

\section{DATA AVAILABILITY STATEMENT}

The datasets analyzed for this study are available on request from GW, gunnar.wichmann@medizin.uni-leipzig.de.

\section{ETHICS STATEMENT}

This study was carried out in accordance with the recommendations of the guidelines of the ethics committee of the Medical 
Faculty of the University Leipzig. The protocol was approved by the ethics committee of the Medical Faculty of the University Leipzig (vote no. 201-10-12072010 and no. 202-10-12072010). All subjects gave written informed consent in accordance with the Declaration of Helsinki.

\section{AUTHOR CONTRIBUTIONS}

GW designed and coordinated the study. $\mathrm{CH}, \mathrm{CL}, \mathrm{SW}, \mathrm{MH}, \mathrm{AD}$, and GW sampled biological specimen from HNSCC patients. Clinical data were provided by $\mathrm{SW}, \mathrm{MH}, \mathrm{AD}$, and GW. $\mathrm{CH}$, $\mathrm{CL}$, and GW performed HLA typing. $\mathrm{CH}$ and GW assessed antigen and haplotype distribution. GW performed statistical analyses and developed the HLA-score. MK and GW discussed statistical models and interpreted the data. GW wrote the first version of the paper. All the authors approved the report.

\section{REFERENCES}

1. Chen Y, Chen C. DNA copy number variation and loss of heterozygosity in relation to recurrence of and survival from head and neck squamous cell carcinoma: a review. Head Neck (2008) 30:1361-83. doi:10.1002/hed.20861

2. Agrawal N, Frederick MJ, Pickering CR, Bettegowda C, Chang K, Li RJ, et al. Exome sequencing of head and neck squamous cell carcinoma reveals inactivating mutations in NOTCH1. Science (2011) 333:1154-7. doi:10.1126/ science. 1206923

3. Stransky N, Egloff AM, Tward AD, Kostic AD, Cibulskis K, Sivachenko A, et al. The mutational landscape of head and neck squamous cell carcinoma. Science (2011) 333:1157-60. doi:10.1126/science.1208130

4. The Cancer Genome Atlas Network. Comprehensive genomic characterization of head and neck squamous cell carcinomas. Nature (2015) 517:576-82. doi:10.1038/nature14129

5. Keck MK, Zuo Z, Khattri A, Stricker TP, Brown C, Imanguli M, et al. Integrative analysis of head and neck cancer identifies two biologically distinct HPV and three non-HPV subtypes. Clin Cancer Res (2014) 21(4):870-81. doi:10.1158/1078-0432.CCR-14-2481

6. Kostareli E, Holzinger D, Bogatyrova O, Hielscher T, Wichmann G, Keck M, et al. HPV-related methylation signature predicts survival in oropharyngeal squamous cell carcinomas. J Clin Invest (2013) 123(6):2488-501. doi:10.1172/ JCI67010

7. Kostareli E, Hielscher T, Zucknick M, Baboci L, Wichmann G, Holzinger D, et al. Gene promoter methylation signature predicts survival of head and neck squamous cell carcinoma patients. Epigenetics (2016) 11(1):61-73. doi:10.108 0/15592294.2015.1137414

8. Wichmann G, Rosolowski M, Krohn K, Kreuz M, Boehm A, Reiche A, et al. Leipzig head and neck group (LHNG). The role of HPV RNA transcription, immune response-related gene expression and disruptive TP53 mutations in diagnostic and prognostic profiling of head and neck cancer. Int J Cancer (2015) 137(12):2846-57. doi:10.1002/ijc.29649

9. Yokoyama A, Muramatsu T, Omori T, Yokoyama T, Matsushita S, Higuchi S, et al. Alcohol and aldehyde dehydrogenase gene polymorphisms and oropharyngolaryngeal, esophageal and stomach cancers in Japanese alcoholics. Carcinogenesis (2001) 22:433-9. doi:10.1093/carcin/22.3.433

10. Rajaee-Behbahani N, Schmezer P, Ramroth H, Bürkle A, Bartsch H, Dietz A, et al. Reduced poly(ADP-ribosyl)ation in lymphocytes of laryngeal cancer patients: results from a case-control study. Int J Cancer (2002) 98:780-4. doi:10.1002/ijc.10234

11. Abbasi R, Ramroth H, Becher H, Dietz A, Schmezer P, Popanda O. Laryngeal cancer risk associated with smoking and alcohol consumption is modified by genetic polymorphisms in ERCC5, ERCC6 and RAD23B but not by polymorphisms in five other nucleotide excision repair genes. Int J Cancer (2009) 125:1431-9. doi:10.1002/ijc.24442

12. Anantharaman D, Marron M, Lagiou P, Samoli E, Ahrens W, Pohlabeln H, et al. Population attributable risk of tobacco and alcohol for upper

\section{ACKNOWLEDGMENTS}

We thank all patients participating in the study and their families, the physicians, documenters, and the technicians involved in execution of the study. We acknowledge support from the German Research Foundation (DFG) and Leipzig University within the program of Open Access Publishing.

\section{FUNDING}

There was no external funding and hence no influence by others on the design of the study, the collection, interpretation, and analysis of the data, the preparation of this report, or decision to publish. GW, CL, $\mathrm{CH}$, and $\mathrm{AD}$ had access to the raw data for the study. The corresponding author had full access to all data and the final responsibility for the decision to submit for publication.

aerodigestive tract cancer. Oral Oncol (2011) 47(8):725-31. doi:10.1016/j. oraloncology.2011.05.004

13. Dietz A, Wichmann G. Head and neck cancer: effective prevention in youth and predictive diagnostics for personalised treatment strategies according to biological differences. EPMAJ(2011) 2:241-9. doi:10.1007/s13167-011-0082-1

14. Golusiński W, Leemans CR, Dietz A. HPV Infection. Head and Neck Cancer. Switzerland: Springer International Publishing (2017).

15. Holzinger D, Flechtenmacher C, Henfling N, Kaden I, Grabe N, Lahrmann B, et al. Identification of oropharyngeal squamous cell carcinomas with active HPV16 involvement by immunohistochemical analysis of the retinoblastoma protein pathway. Int J Cancer (2013) 133(6):1389-99. doi:10.1002/ijc.28142

16. Leemans CR, Snijders PJF, Brakenhoff RH. The molecular landscape of head and neck cancer. Nat Rev Cancer (2018) 18(5):269-82. doi:10.1038/nrc.2018.11

17. Wreesmann VB, Estilo C, Eisele DW, Singh B, Wang SJ. Downregulation of Fanconi anemia genes in sporadic head and neck squamous cell carcinoma. ORL J Otorhinolaryngol Relat Spec (2007) 69:218-25. doi:10.1159/000101542

18. Smith IM, Mithani SK, Mydlarz WK, Chang SS, Califano JA. Inactivation of the tumor suppressor genes causing the hereditary syndromes predisposing to head and neck cancer via promoter hypermethylation in sporadic head and neck cancers. ORL J Otorhinolaryngol Relat Spec (2010) 72:44-50. doi:10.1159/000292104

19. Wichmann G, Herchenhahn C, Boehm A, Mozet C, Hofer M, Fischer M, et al. HLA traits linked to development of head and neck squamous cell carcinoma affect the progression-free survival of patients. Oral Oncol (2017) 69:115-27. doi:10.1016/j.oraloncology.2017.04.017

20. Wichmann G, Krüger A, Boehm A, Kolb M, Hofer M, Fischer M, et al. Induction chemotherapy followed by radiotherapy for larynx preservation in advanced laryngeal and hypopharyngeal cancer: outcome prediction after one cycle induction chemotherapy by a score based on clinical evaluation, computed tomography-based volumetry and ${ }^{18} \mathrm{~F}-\mathrm{FDG}$-PET/CT. Eur J Cancer (2017) 72:144-55. doi:10.1016/j.ejca.2016.11.013

21. Dietz A. DeLOS-II. Randomised Phase II Screening Study To The Use Of A TPFChemotherapy (Short Induction) Before TPF Induction, Radiotherapy With Or Without Cetuximab In The Primary Therapy Of The Only By Laryngectomy Operable Carcinoma of The Larynx/Hypopharynx. EudraCT-Nr.: 2006006091-38. Clinical Trials. Gov, NCT00508664. (2007).

22. Miller SA, Dykes DD, Polesky HF. A simple salting out procedure for extracting DNA from human nucleated cells. Nucleic Acids Res (1988) 16:1215. doi:10.1093/nar/16.3.1215

23. Hanahan D, Weinberg RA. Hallmarks of cancer. The next generation. Cell (2011) 144(5):646-74. doi:10.1016/j.cell.2011.02.013

24. Esteban F, Concha A, Delgado M, Pérez-Ayala M, Ruiz-Cabello F, Garrido F. Lack of MHC class I antigens and tumour aggressiveness of the squamous cell carcinoma of the larynx. Br J Cancer (1990) 62:1047-51. doi:10.1038/ bjc. 1990.437

25. Feenstra M, Veltkamp M, van Kuik J, Wiertsema S, Slootweg P, van den Tweel J, et al. HLA class I expression and chromosomal deletions at $6 p$ and $15 q$ in 
head and neck squamous cell carcinomas. Tissue Antigens (1999) 54:235-45. doi:10.1034/j.1399-0039.1999.540304.x

26. Sidney J, Peters B, Frahm N, Brander C, Sette A. HLA class I supertypes: a revised and updated classification. BMC Immunol (2008) 9:1. doi:10.1186/1471-2172-9-1

27. Khalili JS, Hanson RW, Szallasi Z. In silico prediction of tumor antigens derived from functional missense mutations of the cancer gene census. Oncoimmunology (2012) 1:1281-9. doi:10.4161/onci.21511

28. Russell S, Angell T, Lechner M, Liebertz D, Correa A, Sinha U, et al. Immune cell infiltration patterns and survival in head and neck squamous cell carcinoma. Head Neck Oncol (2013) 5:24.

29. Powers DM. Evaluation: from precision, recall and F-measure to ROC, informedness, markedness and correlation. J Mach Learn Tech (2011) 2: $37-63$.

30. Mattiuz PL, Ihde D, Piazza A, Ceppellini R, Bodmer WF. New approaches to the population genetic and segregation analysis of the HL-A system. In: Terasaki PI, editor. Histocompatibility Testing 1970. Copenhagen: Munksgaard (1970).

31. Machulla HKG, Steinborn F, Tschigrjai M, Langner J, Rainov NG. Meningioma: is there an association with human leukocyte antigens? Cancer Epidemiol Biomarkers Prev (2003) 12:1438-42.
32. Schipper RF, D’Amaro J, de Lange P, Schreuder GM, van Rood JJ, Oudshoorn M. Validation of haplotype frequency estimation methods. Hum Immunol (1998) 59:518-23. doi:10.1016/S0198-8859(98)00054-8

33. Vermorken JB, Stöhlmacher-Williams J, Davidenko I, Licitra L, Winquist E, Villanueva C, et al. SPECTRUM investigators. Cisplatin and fluorouracil with or without panitumumab in patients with recurrent or metastatic squamous-cell carcinoma of the head and neck (SPECTRUM): an open-label phase 3 randomised trial. Lancet Oncol (2013) 14(8):697-710. doi:10.1016/ S1470-2045(13)70181-5

Conflict of Interest Statement: The authors declare that the research was conducted in the absence of any commercial or financial relationships that could be construed as a potential conflict of interest.

Copyright $\odot 2018$ Wichmann, Lehmann, Herchenhahn, Kolb, Hofer, Wiegand and Dietz. This is an open-access article distributed under the terms of the Creative Commons Attribution License (CC BY). The use, distribution or reproduction in other forums is permitted, provided the original author(s) and the copyright owner are credited and that the original publication in this journal is cited, in accordance with accepted academic practice. No use, distribution or reproduction is permitted which does not comply with these terms. 